\title{
Reported Severe Hypersensitivity Reactions after Intravenous Iron Administration in the European Economic Area (EEA) Before and After Implementation of Risk Minimization Measures
}

\author{
Lennart Nathell ${ }^{1} \cdot$ Annegret Gohlke $^{2}$ (D) Stefan Wohlfeil ${ }^{3}$
}

Published online: 3 October 2019

(c) The Author(s) 2019

\begin{abstract}
Introduction Severe hypersensitivity reactions (HSRs) such as anaphylaxis are of great clinical concern because of their life-threatening potential. The adverse events attributable to intravenous iron products include HSRs. An investigation by the European Medicines Agency presented in late 2013 resulted in the implementation of risk minimization measures (RMMs). Objective This study evaluated the number of severe HSRs reported for intravenous iron substances related to exposure for the 4-year periods before and after this implementation.

Methods This was a retrospective pharmacoepidemiologic study with a case-population design. We obtained information from the safety surveillance database EudraVigilance on spontaneously reported severe HSRs using the Medical Dictionary for Regulatory Activities preferred terms "anaphylactic reaction/shock" and "anaphylactoid reaction/shock". Exposure was estimated using IQVIA MIDAS sales data in European economic area countries.

Results Reporting rates for individual products were heterogenous, and the implementation of RMMs appeared to have no clear impact. Reporting rates remained low for the full study period for iron sucrose $(0.03-0.20)$ and ferric gluconate $(0.02-0.14)$ and were higher at the beginning and lower at the end of the study period for ferric carboxymaltose (1.47-0.18). No clear trend was detected for iron dextran (range 0.22-2.80) and iron (III) isomaltoside 1000 (range 0-7.94).

Conclusions Future research is needed to investigate whether the wide variability in reporting rates for severe HSRs associated with these intravenous iron products are due to potential differences in the safety profiles of these substances.
\end{abstract}

\section{Introduction}

Around 1.24 billion individuals worldwide are affected by iron deficiency anemia (IDA), ranking it the fourth-leading cause of disability [1]. Several reasons for IDA exist, including insufficient iron absorption, inadequate dietary iron, blood loss, or an increased physiological requirement for iron. Patients with chronic kidney disease, chronic heart failure, or inflammatory bowel disease are commonly diagnosed

Electronic supplementary material The online version of this article (https://doi.org/10.1007/s40264-019-00868-5) contains supplementary material, which is available to authorized users.

Annegret Gohlke

dr.annegret.gohlke@iqvia.com

1 Nathell Medical AB, Växthusvägen 6, 58276 Linköping, Sweden

2 IQVIA, Landshuter Allee 10, 80637 Munich, Germany

3 Winterthur, Switzerland with IDA [2], and IDA is also associated with other conditions, such as heavy menstruation, acute and chronic gastrointestinal infections affecting iron absorption, and cancer [3].

Adequate supplementation of iron counteracts the adverse effects of iron deficiency. If oral iron replacement is inappropriate because of intolerance, impaired absorption, ongoing bleeding, or nonadherence, intravenous iron therapy is a common alternative treatment [4]. Intravenous iron increases hemoglobin, serum ferritin, and transferrin levels much faster than does oral iron. However, although administration of intravenous iron is generally well-tolerated-the newer iron preparations in particular are generally better tolerated and easier to use than the older preparations-iron infusions can cause hypersensitivity reactions (HSRs) [5, 6], and severe anaphylactic reactions are of great clinical concern [7]. Anaphylaxis is the umbrella term for an acute reaction, defined as a severe, life-threatening generalized or systemic HSR. The diagnosis should be based on clinical symptoms independent of pathomechanisms involved and should distinguish between allergic anaphylaxis and non-immune 


\section{Key Points}

This pharmacoepidemiological study used data from an established pharmacovigilance database and sales data to evaluate the rate of severe hypersensitivity reactions for intravenous iron products before and after the implementation of risk minimization measures.

The results suggest that the overall rate of reported events did not change, but major differences in the reported rates of severe hypersensitivity reactions for individual products were noted.

Future research is needed to elucidate whether the reported differences in the safety profiles of the intravenous iron substances are due to inherent differences in their safety profiles or to impacts of the implemented risk minimization measures.

anaphylaxis (previously classified as anaphylactoid reaction) [8]. Anaphylactic reactions caused by intravenous iron have the potential to be life threatening and so are of special interest to the European health authorities. In 2011, findings were published on differences in HSRs and serious adverse events (AEs; anaphylaxis and other serious allergic AEs) for intravenous iron preparations based on reported rates of anaphylaxis and sales figures [9]. In 2011, a national review by the French National Agency for the Safety of Medicine and Health Products highlighted the risk of serious allergic reactions following administration of intravenous irons, triggering a referral under Article 31 of Directive 2001/83/EC by the European Medicines Agency (EMA) Committee for Medicinal Products for Human Use [5]. In 2013, the EMA referral concluded that the benefit/risk ratio for intravenous iron products on the European market was positive but made no distinction between the individual substance classes (iron sucrose, iron dextran, iron gluconate, ferric carboxymaltose, iron (III) isomaltoside 1000) [5]. As an outcome of the referral, the manufacturers of intravenous iron in the EU were required to conduct a joint post-authorization safety study to characterize hypersensitivity, and retrospective data analysis continues $[5,10]$. The results of this study are expected in $2020[10]$.

To minimize anaphylactic/anaphylactoid reactions, risk minimization measures (RMMs) were implemented, including but not limited to the following:

- no administration during pregnancy unless clearly necessary and then only in the second or third trimester,

- no use in patients with serious hypersensitivity to other parenteral iron products,
- awareness of increased risk in patients with known allergies or immune or inflammatory conditions or with a history of severe asthma, eczema, or other atopic allergy,

- only administered when staff trained to evaluate and manage anaphylactic and anaphylactoid reactions and resuscitation facilities are immediately available [11].

These RMMs are aimed at healthcare professionals, specifically at increasing the understanding and awareness of the risk of severe HSRs and how to manage them. The implementation of RMMs was aligned by the market authorization holders in the respective European countries and in accordance with the timelines stipulated in the referral assessment report [12]. A direct healthcare professional communication was sent out within 30 days of the European Commission decision, and education material for prescribers and patients, highlighting the risks of and warnings about HSRs, was sent within 3 months.

In 2015, another study in the USA compared the risk of anaphylactic reactions for several intravenous iron products. The authors found that the risk was highest for iron dextran, followed in decreasing order by ferumoxytol, ferric gluconate, and iron sucrose [13]. This study did not include ferric carboxymaltose or iron (III) isomaltoside 1000 because they were not available in the US during the study period (2003-2013). Also in 2015, a report by the Netherlands Pharmacovigilance Centre Lareb stated that they had received several notifications of concern from multiple Dutch hospitals about an increase in the frequency and severity of allergic reactions to intravenous iron-containing medicines after a product switch from iron carboxymaltose to iron (III) isomaltoside 1000 [14]. A 2017 retrospective analysis of a patient cohort that included all patients receiving intravenous iron infusions in an outpatient gastroenterology setting found that the risk of HSRs was fourfold higher with iron (III) isomaltoside 1000 than with ferric carboxymaltose [15]. Similarly, a 2018 single-center cohort study from the Netherlands reported that the likelihood of an HSR was 3.4 times higher with iron isomaltoside 1000 than with ferric carboxymaltose [16]. In addition, reports of fatal anaphylactic reactions to iron (III) isomaltoside 1000 in a Spanish region in 2017 led to regulatory activities from the Spanish Agency of Medicines and Medical Products, including a recommendation that no new treatment with iron (III) isomaltoside 1000 be started [17].

To further investigate potential differences in the reporting of severe HSRs, we recently conducted a pharmacoepidemiologic database study [18] using a case-population design to assess the number of anaphylactic/anaphylactoid reactions reported following administration of the two most commonly used high-dose intravenous iron products (ferric carboxymaltose and iron (III) isomaltoside 1000) in relation to exposure in European countries between 2014 and 2017 
(EU PAS Register Number EUPAS25192). The results suggested that iron (III) isomaltoside 1000 was associated with more reports of severe HSRs related to estimated exposure than was ferric(III) carboxymaltose 1000 [18].

To evaluate the impact of RMMs on the overall and substance-specific reporting rates of severe HSRs associated with intravenous iron-containing substances with respect to overall exposure of each substance for the 4-year periods before (2010-2013) and after (2014-2017) implementation of these measures, this study was conducted with the same case-population design from 2010 to 2017 and included all intravenous iron-containing products in European economic area (EEA) countries. A further objective was to identify trends in the reporting rates of HSRs for each intravenous iron substance over the complete period (2010-2017).

\section{Methods}

\subsection{Study Design}

This was a retrospective pharmacoepidemiologic study with a case-population design [19]. The study was conducted for EEA countries for the study period 1 January 2010-31 December 2017. This period was chosen to specifically include the two 4-year periods before and after the conclusions of the EMA referral on intravenous iron-containing medicinal products were presented (September 2013) and actions were taken [5].

\subsection{Variables and Corresponding Data Sources}

We obtained information on the number of spontaneous reports of severe HSRs in EEA countries from the established centralized European safety surveillance database EudraVigilance [20]. We chose this source for this retrospective analysis because it systematically collects all safety data reported by pharmaceutical companies and national competent authorities in EEA countries. HSRs are generally serious AEs, but their severity grades vary. To focus on the more severe reactions with substantial consequences, including fatality, we narrowed the search to severe reactions. For this and our previous study [18], spontaneous reports of severe HSRs were identified using the Medical Dictionary for Regulatory Activities (MedDRA ${ }^{\circledR}$ ) preferred terms "anaphylactic reaction, anaphylactic shock, anaphylactoid reaction, and anaphylactoid shock" associated with administration of iron sucrose, ferric carboxymaltose, ferric gluconate, ferumoxytol, iron dextran, or iron (III) isomaltoside 1000 . Reports cannot be attributed to individual countries but were obtained for the EEA as a whole.

We estimated exposure to intravenous iron substances using sales data for these products in EEA countries collected via the IQVIA MIDAS platform. For most countries, sales are captured for both hospital and retail settings. IQVIA MIDAS sales data coverage varies by country and setting but is high for most countries, ranging from 75 to $100 \%$ for $80 \%$ of the countries of interest and to $>95 \%$ for more than $60 \%$ of countries. For countries where coverage was not $100 \%$, figures were projected to the country level because coverage was large enough to allow extrapolation to the entire country, even for those with coverage $<50 \%$. For our analysis, we assumed that sales data accurately reflected the use of and exposure to each substance (i.e., inventory levels do not vary across the 8 -year period as hospital stocks are usually kept low for economic reasons). Several studies each year validate the quality and accuracy of the sales data, and a global precision index was published with a value of $94.3 \%$ in 2017 [21]. Sales data were available for 27 EEA countries (Austria, Belgium, Bulgaria, Croatia, Czech Republic, Denmark, Estonia, Finland, France, Germany, Greece, Hungary, Ireland, Italy, Latvia, Lithuania, Luxembourg, The Netherlands, Norway, Poland, Portugal, Romania, Slovakia, Slovenia, Spain, Sweden, and the UK). Sales were normalized to $100-\mathrm{mg}$ dose equivalents (DEq; $100 \mathrm{mg}$ $\mathrm{DEq}$ of iron $=$ one defined daily dose [DDD]) of iron. This is in accordance with a previously published method of standardization [22].

\subsection{Analysis}

The statistical analysis was conducted descriptively. Reports on anaphylactic reactions, anaphylactic shock, anaphylactoid reactions, and anaphylactoid shock were summed and presented under the term "severe HSRs".

Overall reporting rates were determined by dividing the number of reports of severe HSRs by the corresponding sales volume of $100 \mathrm{mg}$ DEq of iron for each class of intravenous iron in all 27 countries (see Sect. 2.2). This was also performed for the total class of intravenous iron. Given the impossibility of determining the exact dose administered to each patient, we applied the same approach as Bailie et al. $[9,22]$, who arbitrarily attributed the same average dose to all groups of patients. Doses were standardized to $100 \mathrm{mg}$ DEq of iron [9, 22]. Reporting rates were calculated for each substance, for total class by year, and per period. A twosided 95\% confidence interval (CI) assuming normal distribution was considered as a default $(\alpha=5 \%) .95 \%$ CI for reporting rate $(95 \%)=\hat{p} \pm z \times \sqrt{\frac{\hat{p}(1-\hat{p})}{n}}$; where $\hat{p}=$ sample proportion, $n=$ sample size, and $z=1.96$.

We conducted a sensitivity analysis including only sales data from countries with at least three reports for any reaction to account for countries in the EEA with a high rate of underreporting. 


\section{Results}

\subsection{Reported Number of Severe Hypersensitivity Reactions (HSRs) in EudraVigilance}

From 2010 to 2017 , a total of 55 and 144 events were reported for iron sucrose and ferric carboxymaltose, respectively, in the EEA. In the same period and region, 37 events were reported for ferric gluconate, one event was reported for ferumoxytol, 77 for iron dextran, and 177 events for iron (III) isomaltoside 1000 (Table 1).

Two cases of anaphylactic/anaphylactoid reaction resulting in death were reported for ferric carboxymaltose, two for iron dextran, one for iron (III) isomaltoside 1000, and none for iron sucrose, ferric gluconate, and ferumoxytol.

From 2010 to 2017, the number of events per year remained in the same range for iron sucrose (2-11), ferric carboxymaltose (12-25), ferric gluconate (1-8), and ferumoxytol (0-1). Only one report was submitted for ferumoxytol in 2015. Reported events for iron dextran were higher in 2010 and 2011 (21-22) then decreased to 1-11 events per year in the period 2012-2017. For iron (III) isomaltoside 1000 , numbers were lower in the first 3 years $(0-8$ events from 2010 to 2012 vs. 24-46 events from 2013 to 2017). As sales were very low for ferumoxytol, results for this substance are not depicted in further tables and figures.

\subsection{Exposure to Intravenous Irons Based on Sales Data}

In the EEA, the annual total exposure to iron sucrose and iron sucrose similars was stable across the years, ranging from 5.4 to 5.9 million DDDs. Annual total exposure to ferric carboxymaltose steadily increased from 0.8 million DDDs in 2010 to approximately 10.6 million in 2017. Exposure to ferric gluconate during this period ranged from 6.8 to 4.9 million DDDs between 2010 and 2017, respectively.
Exposure to ferumoxytol was very low, ranging from $<0.01$ in 2012 to 0.01 in 2014. Ferumoxytol was launched in the EEA in 2012 but was taken off the market in 2015. Exposure to iron dextran, as to iron sucrose, was also stable, ranging from 0.5 to 0.8 million DDDs in the same time period. Exposure to iron (III) isomaltoside 1000 increased from $<0.01$ to 1.4 million DDDs for the same period (Fig. 1).

\subsection{Characteristics of Patients with Reported Severe HSRs}

In EudraVigilance, data on age group and sex were available for most patients with a reported severe HSR: iron sucrose, $N=45$ of 55 ; ferric carboxymaltose, $N=118$ of 144; ferric gluconate, $N=30$ of 37 ; ferumoxytol, $N=1$ of 1 ; iron dextran, $N=68$ of 77 ; iron (III) isomaltoside $1000, N=165$ of 177. The following percentages of reported severe HSRs occurred in female patients: $68.9 \%(N=31$ of 45$)$ for iron sucrose, $86.4 \%$ ( $N=102$ of 118$)$ for ferric carboxymaltose, 93.3\% ( $N=28$ of 30) for ferric gluconate, $100 \%(N=1$ of 1) for ferumoxytol, $76.5 \%(N=52$ of 68$)$ for iron dextran, and $63.6 \%(N=105$ of 165$)$ for iron (III) isomaltoside 1000 .

The vast majority of patients with reported severe HSRs in EudraVigilance were aged 18-64 years (ferric carboxymaltose, $78.8 \%$; ferric gluconate, $76.7 \%$; iron (III) isomaltoside $1000,71.5 \%$ ). Only half of the reported severe HSRs occurred in patients in this age group for iron sucrose $(48.9 \%)$ and iron dextran (55.9\%).

More than half of the reports $(N=292$ [59.5\%]) included information on dosing. Of these, median \pm standard deviation (SD) doses were $300 \pm 306 \mathrm{mg}$ for patients receiving iron sucrose, $1000 \pm 347 \mathrm{mg}$ for the 97 patients receiving ferric carboxymaltose, $63 \pm 75 \mathrm{mg}$ for those receiving ferric gluconate, $100 \pm 517 \mathrm{mg}$ for iron dextran, and $1000 \pm 389 \mathrm{mg}$ of iron (III) isomaltoside 1000.
Table 1 Reported number of severe hypersensitivity reactions with intravenous iron products in European economic area countries per year

\begin{tabular}{lccllcc}
\hline Year & Iron sucrose & $\begin{array}{l}\text { Ferric car- } \\
\text { boxymaltose }\end{array}$ & $\begin{array}{l}\text { Ferric } \\
\text { gluconate }\end{array}$ & Ferumoxytol & Iron dextran & $\begin{array}{l}\text { Iron (III) } \\
\text { isomaltoside } \\
1000\end{array}$ \\
\hline Total & 55 & 144 & 37 & 1 & 77 & 177 \\
2010 & 8 & 12 & 8 & 0 & 21 & 0 \\
2011 & 2 & 17 & 4 & 0 & 22 & 1 \\
2012 & 6 & 14 & 3 & 0 & 7 & 8 \\
2013 & 11 & 25 & 1 & 0 & 11 & 31 \\
2014 & 7 & 15 & 7 & 0 & 8 & 24 \\
2015 & 8 & 18 & 4 & 1 & 1 & 41 \\
2016 & 8 & 24 & 3 & 0 & 2 & 46 \\
2017 & 5 & 19 & 7 & 0 & 5 & 26 \\
\hline
\end{tabular}




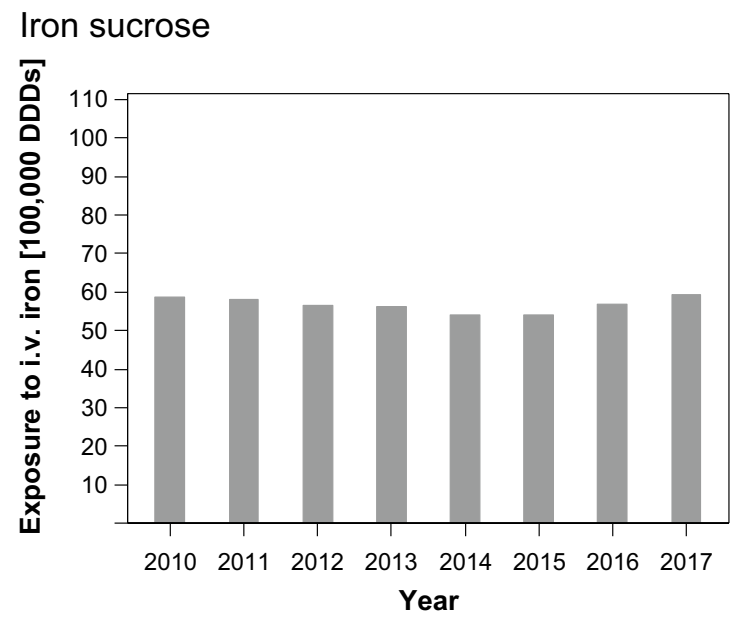

Ferric gluconate

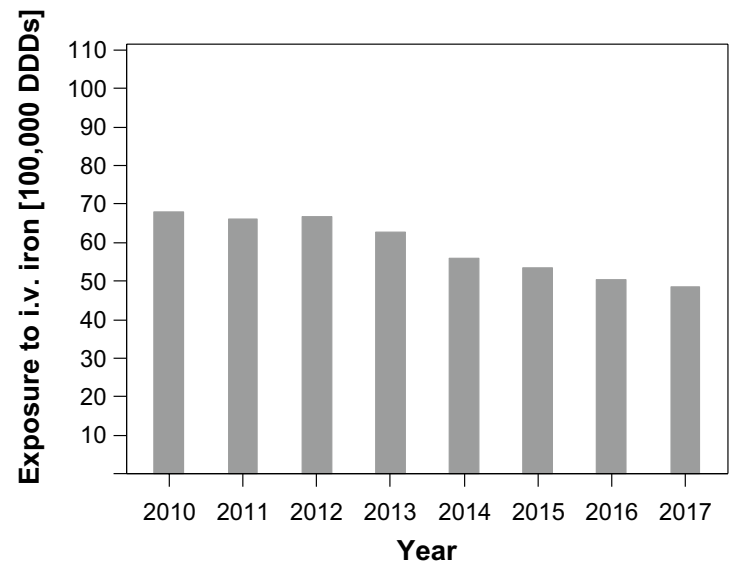

Iron dextran

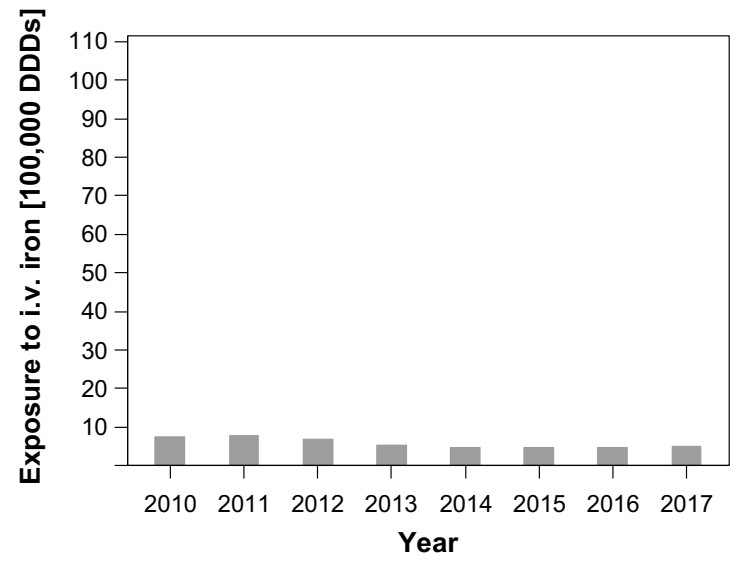

Ferric carboxymaltose

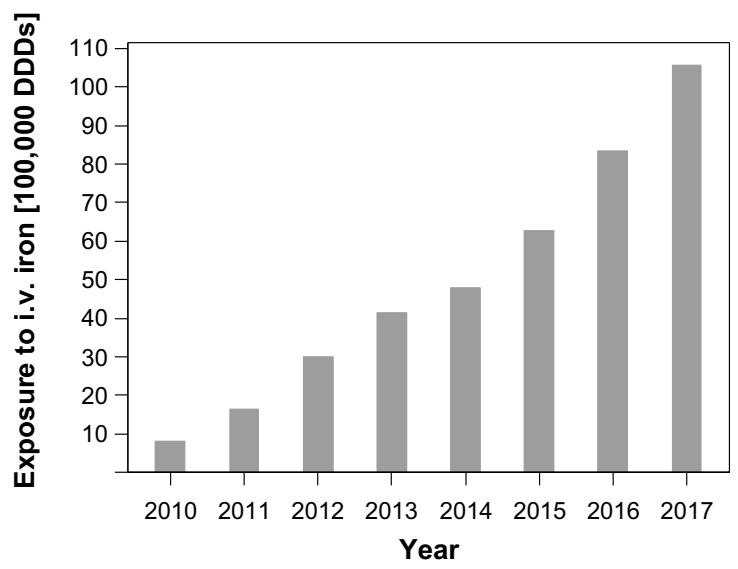

\section{Ferumoxytol}

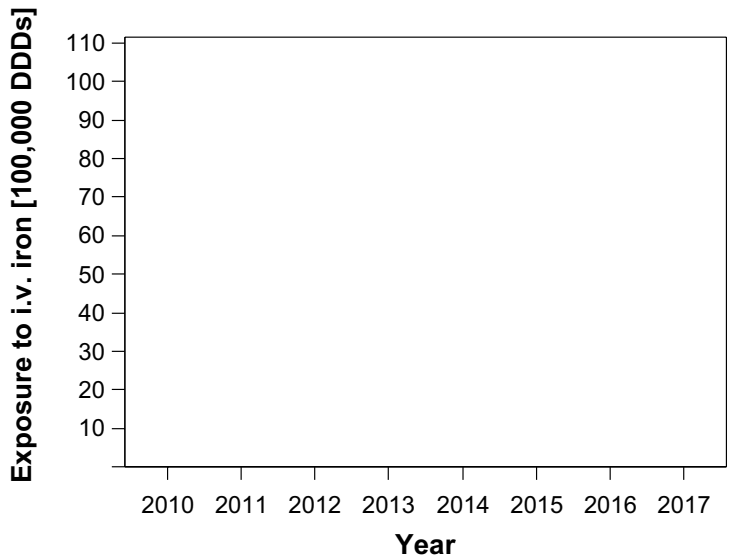

Iron (III) isomaltoside 1000

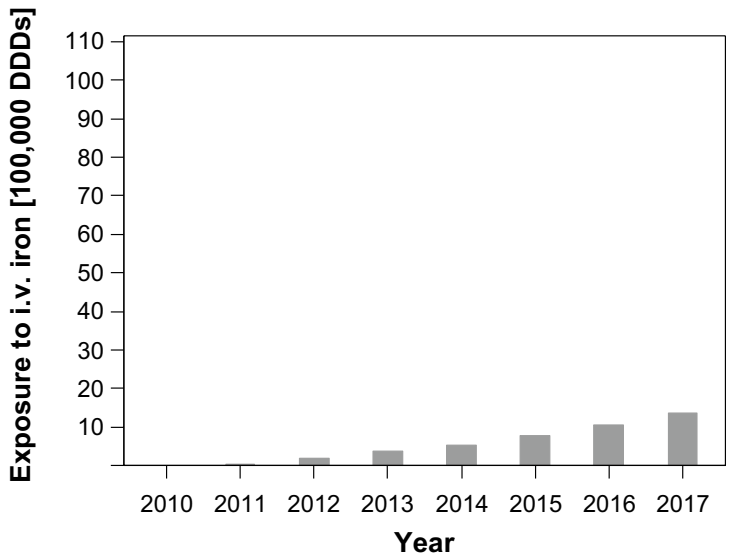

Fig. 1 Exposure to intravenous iron products in European economic area countries from 2010 to 2017. $D D D$ defined daily dose, i.v. intravenous

\subsection{Reporting Rates for Severe HSRs}

Between 2010 and 2017, reporting rates varied from 0.03 (95\% CI -0.01 to 0.08 ) to 0.20 (95\% CI $0.08-0.31$ ) for iron sucrose, from 0.18 (95\% CI $0.10-0.26)$ to 1.47 (95\%
CI 0.64-2.30) for ferric carboxymaltose, from 0.02 (95\% CI -0.02 to 0.05$)$ to 0.14 (95\% CI $0.04-0.25)$ for ferric gluconate, from 0.22 (95\% CI -0.21 to 0.64$)$ to 2.80 (95\% CI 1.63-3.97) for iron dextran, and from 0 to 7.94 (95\% CI 5.15-10.74) for iron (III) isomaltoside 1000 (see Fig. 2; 
Fig. 2 Reporting rates of severe hypersensitivity reactions (anaphylactic/anaphylactoid reactions) per 100,000 DDDs of intravenous irons in the EudraVigilance database excluding ferumoxytol. $A E$ adverse event, $D D D$ defined daily dose

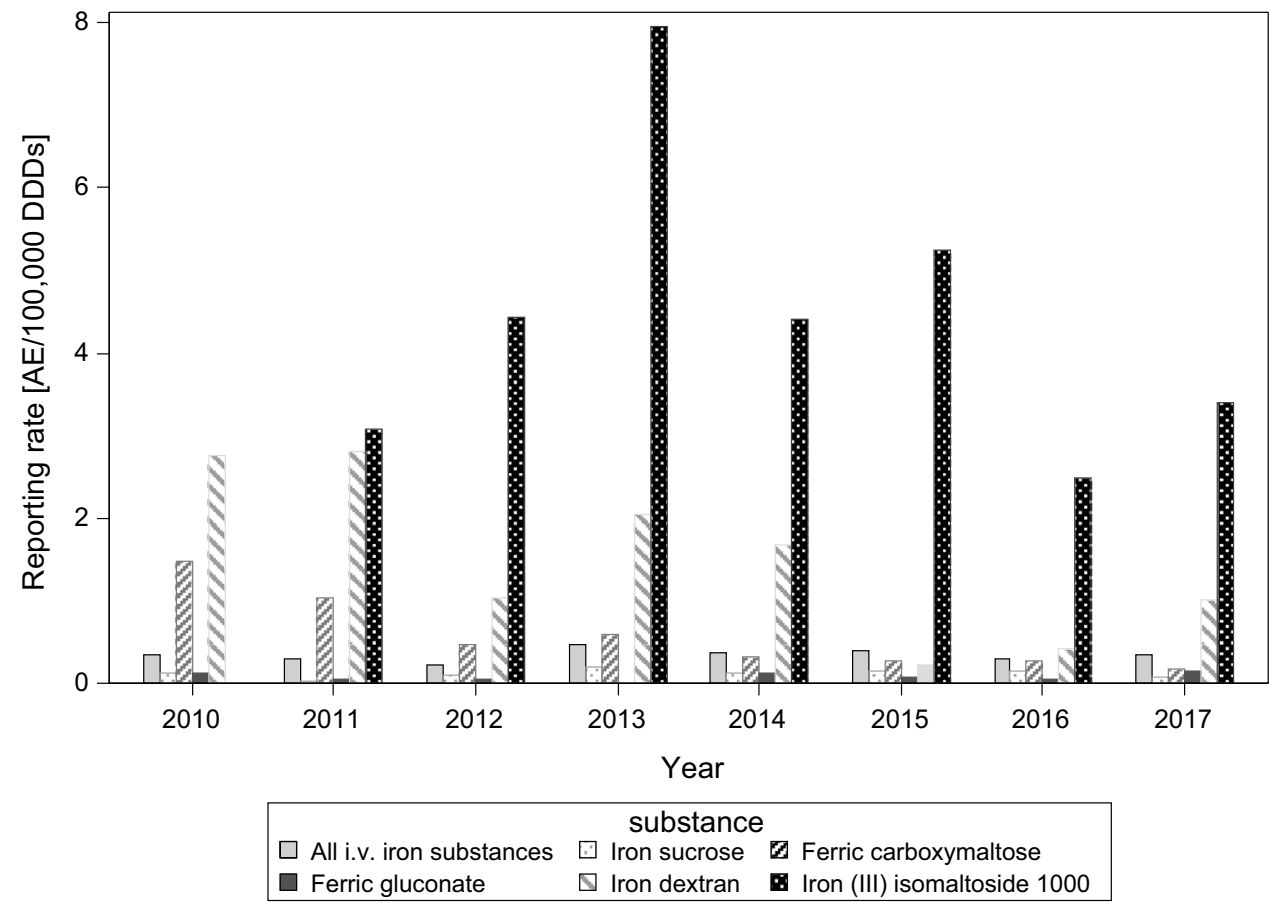

Table 2 Reporting rate of severe hypersensitivity reactions for intravenous iron substances by year and period

\begin{tabular}{lllllll}
\hline Year/period & $\begin{array}{l}\text { All IV iron sub- } \\
\text { stances }\end{array}$ & Iron sucrose & $\begin{array}{l}\text { Ferric carboxy- } \\
\text { maltose }\end{array}$ & Ferric gluconate & Iron dextran & $\begin{array}{l}\text { Iron (III) isomalto- } \\
\text { side } 1000\end{array}$ \\
\hline Pre $(2010-2013)$ & $0.34(0.29 ; 0.39)$ & $0.12(0.07 ; 0.16)$ & $0.71(0.54 ; 0.88)$ & $0.06(0.03 ; 0.09)$ & $2.21(1.66 ; 2.77)$ & $6.63(4.57 ; 8.68)$ \\
Post $(2014-2017)$ & $0.35(0.31 ; 0.40)$ & $0.12(0.08 ; 0.17)$ & $0.25(0.20 ; 0.31)$ & $0.10(0.06 ; 0.14)$ & $0.85(0.43 ; 1.26)$ & $3.68(3.06 ; 4.30)$ \\
2010 & $0.34(0.25 ; 0.44)$ & $0.14(0.04 ; 0.23)$ & $1.47(0.64 ; 2.30)$ & $0.12(0.04 ; 0.20)$ & $2.77(1.59 ; 3.96)$ & 0 \\
2011 & $0.31(0.22 ; 0.40)$ & $0.03(-0.01 ; 0.08)$ & $1.03(0.54 ; 1.52)$ & $0.06(0.00 ; 0.12)$ & $2.80(1.63 ; 3.97)$ & $3.09(-2.97 ; 9.15)$ \\
2012 & $0.23(0.16 ; 0.31)$ & $0.11(0.02 ; 0.19)$ & $0.47(0.22 ; 0.71)$ & $0.05(-0.01 ; 0.10)$ & $1.03(0.27 ; 1.79)$ & $4.43(1.36 ; 7.50)$ \\
2013 & $0.47(0.36 ; 0.57)$ & $0.20(0.08 ; 0.31)$ & $0.60(0.37 ; 0.84)$ & $0.02(-0.02 ; 0.05)$ & $2.06(0.84 ; 3.27)$ & $7.94(5.15 ; 10.74)$ \\
2014 & $0.36(0.27 ; 0.45)$ & $0.13(0.03 ; 0.23)$ & $0.31(0.15 ; 0.47)$ & $0.13(0.03 ; 0.22)$ & $1.68(0.52 ; 2.84)$ & $4.40(2.64 ; 6.16)$ \\
2015 & $0.40(0.31 ; 0.49)$ & $0.15(0.05 ; 0.25)$ & $0.29(0.15 ; 0.42)$ & $0.07(0.00 ; 0.15)$ & $0.22(-0.21 ; 0.64)$ & $5.25(3.64 ; 6.85)$ \\
2016 & $0.31(0.23 ; 0.38)$ & $0.14(0.04 ; 0.24)$ & $0.29(0.17 ; 0.40)$ & $0.06(-0.01 ; 0.13)$ & $0.43(-0.17 ; 1.03)$ & $2.49(1.53 ; 3.45)$ \\
2017 & $0.35(0.28 ; 0.43)$ & $0.08(0.01 ; 0.16)$ & $0.18(0.10 ; 0.26)$ & $0.14(0.04 ; 0.25)$ & $1.03(0.13 ; 1.92)$ & $3.40(2.42 ; 4.39)$ \\
\hline
\end{tabular}

Severe hypersensitivity reactions per 100,000 defined daily doses ( $95 \%$ confidence interval)

$I V$ intravenous

Table 2). Ferumoxytol was excluded from the analysis because it was only on the market from 2012 to 2015 . To account for EEA countries with a high rate of underreporting to EudraVigilance, we considered only exposure data from countries that reported more than three adverse reactions (any adverse reaction, not limited to HSRs or related to ironcontaining products) to EudraVigilance in the denominator for the sensitivity analysis. The results of this sensitivity analysis supported those from the primary analysis (data not shown).
Table 2 lists the overall and substance-specific reporting rates for the two 4-year periods before and after implantation of the RMMs.

\section{Discussion}

We evaluated the impact of RMMs on the overall and substance-specific reporting rates for HSRs associated with intravenous iron substances with respect to overall exposure of each. Furthermore, our findings describe reporting rate trends between 2010 and 2017 for intravenous iron 
products for all EEA countries. This study is a follow-up to our recently published real-world database study [18], extending the list of substances to all intravenous irons and including the years before and after the referral in 2013. So far, no comparative safety study on severe HSRs associated with all of these intravenous irons has been published; thus, these real-world data, from an established source of information on the safety profile of drugs in clinical practice, provide valuable insights.

As in our previous study, most severe HSRs were reported for female patients. This reflects the higher usage of intravenous iron in females, with pregnancy and menstruation leading to a higher prevalence of anemia in women than in men [23].

Overall, we observed that reporting rates for the intravenous iron products were heterogenous. For iron sucrose and ferric gluconate, the reporting rates remained at a similar level from 2010 to 2017, confirming previous reports on the established safety profile for these drugs [9, 13]. For ferric carboxymaltose and iron dextran, the reporting rates were higher at the beginning and lower at the end of this period, whereas the reporting rate for iron (III) isomaltoside 1000 was higher in the middle than at the beginning and lower again at the end of the period. This might indicate that the RMMs had a higher impact for ferric carboxymaltose and iron dextran than for the other products.

An investigation of time trends indicated that the reporting of adverse drug reactions is expected to be higher directly after launch than when products are well-established on the market or may be influenced by and expected to be higher directly after a public discussion on safety concerns [24]. However, capturing the period before (2010-2013) and after the referral (2014-2017), the reporting rate was similar for iron sucrose and iron gluconate/ferric gluconate in both periods, whereas the reporting rate for ferric carboxymaltose, iron (III) isomaltoside 1000, and iron dextran was lower in the post-referral period than in the pre-referral period.

When looking at the numbers before and after referral in isolation, it seems the implemented RMMs did in fact have an impact on reporting. However, investigation of data from individual years shows that this is a time-dependent effect over the full period, with wide variability and no striking difference between the periods before and after RMM implementation attributable to the referral or the RMMs. Initially, this might seem surprising, but a closer look at the RMMs reveals reasons for this: most measures were aimed at safety procedures after HSRs occur, e.g., intravenous iron should only be administered when resuscitation facilities and staff trained to evaluate and manage anaphylactic and anaphylactoid reactions are immediately available. Other RMMs aimed at preventing HSRs might already have been followed before the implementation of RMMs, e.g., no administration of intravenous irons during pregnancy, no use in patients with serious hypersensitivity to other parenteral iron products, and awareness of increased risk in patients with known risk factors, such as allergies, immune or inflammatory conditions, or history of severe asthma, eczema, or other atopic allergy.

In addition to the expected increase in reporting following a public discussion of safety concerns, reporting of suspected adverse drug reactions may be higher directly after launch than when products are well-established on the market (so-called Weber effect). Since both established and products newly launched in several countries were included in the analysis, an impact on the reporting rate cannot be ruled out. Ferric carboxymaltose and iron (III) isomaltoside 1000 were launched only a short time before the study period of 2010-2017 (ferric carboxymaltose in June 2007 and iron (III) isomaltoside 1000 in December 2009; see Table S1 in the Electronic Supplementary Material [ESM]), so the Weber effect is likely for these two substances, especially for the pre-referral period before 2014. Conversely, iron sucrose, iron dextran, and iron gluconate/ferric gluconate have been on the European market for longer (iron sucrose since 1949, ferric gluconate since the 1960s, low-molecular-weight iron dextran since 2000). Ferumoxytol was on the market for only a limited time overall, making a comparison infeasible. Furthermore, reporting levels for suspected severe HSRs may also vary in different healthcare systems. However, there is a notable difference between the intravenous iron compounds, with iron sucrose and ferric gluconate showing the lowest rates and iron isomaltoside the highest, both before and after the RMMs. Furthermore, the magnitude of difference in the frequency of reported severe HSRs adds validity to our results. Another major difference between the intravenous iron products included in this analysis is the carbohydrate shell, which leads to different physico-chemical properties and could explain the difference in severe HSR reporting rates (see Table $\mathrm{S} 1$ in the ESM).

As in our previous study [18], the results of this pharmacoepidemiological study analyzing spontaneous reports have known limitations, such as the use of different preferred MedDRA ${ }^{\circledR}$ terms for AEs, changes in AE coding over time, inconsistent descriptions of AEs by clinicians, no confirmation of event term or causal relationship for AEs reported by patients, and different reports in national and international surveillance databases. As these general issues apply for all substances, we believe that the impact on a comparison of substances is likely minor. Furthermore, underreporting of AEs to spontaneous reporting systems is well-known [25]. However, our analysis focused on severe events where underreporting is less likely, as practitioners continue to report serious adverse drug reactions after the initial years of marketing [24]. It is also worth noting that recent research has challenged the existence of a Weber effect, originally described in 1984 [26, 27]. 
With respect to the estimated exposure based on sales data, the following considerations should also be addressed. The MIDAS database does not capture all sales data, such as direct sales to clinics and private offices within each country. In some countries, not all distribution channels (hospital/ retail) are captured in the MIDAS database. For example, hospital data are not available for Estonia, Greece, and Latvia. Furthermore, availability of the substances varies across countries, which might affect the respective reporting rates.

Reporting rates should be compared between different iron-containing products with caution because the true average dose (especially for high-dose products) might be substantially higher, by the same factor it differs from the common DDD definition ( $1 \mathrm{DDD}=100 \mathrm{mg})$. In addition, the underlying treated population for each substance might differ by important potential confounders, such as indicated disease, comorbidities, and age. Although no direct effect of these characteristics on the outcome of severe HSRs is described in the literature, it might impact the number of AEs reported by healthcare providers. Nevertheless, these results align with those in previous publications on the safety profile of iron sucrose [9, 13], which provides some reassurance. An interrupted time series (ITS) analyzing the reporting rates before and after RMM implementation would have strengthened our results, and we assessed the feasibility of conducting an ITS (for the entirety of products and for each substance separately) using the current datasets. The small number of time points (four before and four after RMM implementation) and the wide variability in both the period before and the period after RMM implementation meant that the statistical model did not converge in any ITS analysis for any of the substances. A statistical test simply comparing pre- and post-implementation rates (with the mean rate of the four time points considered for each period) is considered inappropriate because it does not account for possible trends over time.

Reporting of AEs does not necessarily reflect the occurrence of events in clinical practice, so these results preclude any conclusions about the absolute and relative risk for severe HSRs associated with the intravenous irons presented. Although AE reporting can be used to estimate the relative rates of events with individual products, head-to-head data remain the gold standard because they capture exposure and outcome in a standardized manner and circumvent the effects of differential prescribing and reporting.

It is also important to consider that the results provide a description of reporting rates for intravenous iron-containing products before and after the referral but are not suitable to assess the effectiveness of the actual RMMs that were introduced after the referral in EEA countries.

\section{Conclusion}

No clear change in the reporting rates of severe HSRs (i.e., anaphylactic/anaphylactoid reactions) was associated with intravenous iron-containing products for EEA countries following the implementation of RMMs in 2013. Future research is needed to further elucidate whether the reported differences in the safety profiles of the intravenous iron substances are due to inherent differences in their safety profiles or to different impacts of the RMMs.

Acknowledgements The authors thank Dorothea von Bredow for reviewing the manuscript and providing input.

\section{Compliance with ethical standards}

Funding This study was funded by Vifor Pharma International AG.

Conflicts of interest Stefan Wohlfeil was an employee of Vifor Pharma Management Ltd during the planning and execution of the study and holds shares in Vifor Pharma. Lennart Nathell is a former employee of Vifor Pharma; during the planning, execution, and publishing of the study Lennart worked for a company on a contract with Vifor Pharma Management Ltd. Vifor Pharma is the manufacturer of Ferinject ${ }^{\circledR}$ (ferric carboxymaltose) and Venofer ${ }^{\circledR}$ (iron sucrose). Annegret Gohlke is employee of IQVIA, which received funding from Vifor Pharma International AG for the conduct of the study.

Data Availability Reports on anaphylactic reactions, anaphylactic shocks, anaphylactoid reactions, and anaphylactoid shocks can be publicly accessed via EudraVigilance: https://www.ema.europa.eu/en/ human-regulatory/research-development/pharmacovigilance/eudravigil ance/access-eudravigilance-data.

Disclaimer Data derived from EudraVigilance: The views expressed in this article are the personal views of the author(s) and may not be understood or quoted as being made on behalf of or reflecting the position of the EMA or one of its committees or working parties.

Open Access This article is distributed under the terms of the Creative Commons Attribution-NonCommercial 4.0 International License (http://creativecommons.org/licenses/by-nc/4.0/), which permits any noncommercial use, distribution, and reproduction in any medium, provided you give appropriate credit to the original author(s) and the source, provide a link to the Creative Commons license, and indicate if changes were made.

\section{References}

1. Vos T, Abajobir AA, Abate KH, Abbafati C, Abbas KM, AbdAllah F, et al. Global, regional, and national incidence, prevalence, and years lived with disability for 328 diseases and injuries for 195 countries, 1990-2016: a systematic analysis for the Global Burden of Disease Study 2016. Lancet. 2017;390:1211-59. https ://doi.org/10.1016/S0140-6736(17)32154-2.

2. Cappellini MD, Comin-Colet J, de Francisco A, Dignass A, Doehner W, Lam CS, et al. Iron deficiency across chronic inflammatory conditions: international expert opinion on definition, diagnosis, 
and management. Am J Hematol. 2017;92:1068-78. https://doi. org/10.1002/ajh.24820.

3. WHO/CDC. Worldwide prevalence of anaemia 1993-2005: WHO Global Database on Anemia. Geneva: WHO; 2008.

4. Bhandari S, Pereira DIA, Chappell HF, Drakesmith H. Intravenous irons: from basic science to clinical practice. Pharmaceuticals (Basel). 2018. https://doi.org/10.3390/ph11030082.

5. EMA CHMP. Assessment report for: Iron containing intravenous (IV) medicinal products EMA/549569/2013. 2013. http://www. ema.europa.eu/docs/en_GB/document_library/Referrals_docum ent/IV_iron_31/WC500150771.pdf. Accessed 8 Aug 2018.

6. Hussain I, Bhoyroo J, Butcher A, Koch TA, He A, Bregman DB. Direct comparison of the safety and efficacy of ferric carboxymaltose versus iron dextran in patients with iron deficiency anemia. Anemia. 2013;2013:169107. https://doi.org/10.1155/2013/16910 7.

7. Kidney Disease: Improving Global Outcomes. KDIGO clinical practice guideline for anemia in chronic kidney disease. Kidney Int Suppl. 2012;2:280-335.

8. Johansson SGO, Bieber T, Dahl R, Friedmann PS, Lanier BQ, Lockey RF, et al. Revised nomenclature for allergy for global use: report of the Nomenclature Review Committee of the World Allergy Organization, October 2003. J Allergy Clin Immunol. 2004;113:832-6. https://doi.org/10.1016/j.jaci.2003.12.591.

9. Bailie GR, Horl WH, Verhoef J-J. Differences in spontaneously reported hypersensitivity and serious adverse events for intravenous iron preparations: comparison of Europe and North America. Arzneimittelforschung. 2011;61:267-75. https://doi. org/10.1055/s-0031-1296198.

10. encepp.eu. Intravenous Iron Postauthorisation Safety Study (PASS): evaluation of the risk of severe hypersensitivity reactions. 2018. http://www.encepp.eu/encepp/viewResour ce.htm?id=24010. Accessed 1 Oct 2018.

11. EMA CHMP. New recommendations to manage risk of allergic reactions with intravenous iron-containing medicines. 2013. https ://www.ema.europa.eu/documents/referral/intravenous-iron-conta ining-medicinal-products-article-31-referral-new-recommenda tions-manage-risk_en.pdf. Accessed 25 Jan 2019.

12. EMA CHMP. Annex IV conditions to the marketing authorisations. 2013. https://www.ema.europa.eu/en/documents/referral/ intravenous-iron-containing-medicinal-products-article-31-refer ral-annex-iv_en.pdf. Accessed 17 Sep 2019.

13. Wang C, Graham DJ, Kane RC, Xie D, Wernecke M, Levenson M, et al. Comparative risk of anaphylactic reactions associated with intravenous iron products. JAMA. 2015;314:2062-8. https://doi. org/10.1001/jama.2015.15572.

14. Bijwerkingen Centrum Lareb. Intravenous iron preparations and allergic reactions. 2015. https://databankws.lareb.nl/Downloads/ Signals_2015_Intravenous_iron.pdf. Accessed 7 May 2018.

15. Bager P, Hvas CL, Dahlerup JF. Drug-specific hypophosphatemia and hypersensitivity reactions following different intravenous iron infusions. Br J Clin Pharmacol. 2017;83:1118-25. https://doi. org/10.1111/bcp.13189.
16. Mulder MB, van den Hoek, Hester L, Birnie E, van Tilburg, Antonie JP, Westerman EM. Comparison of hypersensitivity reactions of intravenous iron: iron isomaltoside-1000 (Monofer((R))) versus ferric carboxy-maltose (Ferinject $((\mathrm{R})))$. A single center, cohort study. Br J Clin Pharmacol. 2019;85:385-92. https://doi. org/10.1111/bcp.13805.

17. WHO Press. Pharmaceuticals Newsletter. Geneva: WHO Press; 2017.

18. Ehlken B, Nathell L, Gohlke A, Bocuk D, Toussi M, Wohlfeil $\mathrm{S}$. Evaluation of the reported rates of severe hypersensitivity reactions associated with ferric carboxymaltose and iron(III) isomaltoside 1000 in europe based on data from EudraVigilance and VigiBase $^{\mathrm{TM}}$ between 2014 and 2017. Drug Saf. 2019;42:463-71. https://doi.org/10.1007/s40264-018-0769-5.

19. Theophile H, Laporte J-R, Moore N, Martin K-L, Begaud B. The case-population study design: an analysis of its application in pharmacovigilance. Drug Saf. 2011;34:861-8. https://doi. org/10.2165/11592140-000000000-00000.

20. European Medicines Agency. EudraVigilance system overview. https://www.ema.europa.eu/en/human-regulatory/research-devel opment/pharmacovigilance/eudravigilance/eudravigilance-syste m-overview. Accessed 27 Nov 2018.

21. IQVIA Commercial GmbH \& Co. OHG. acts 2018. 2019. https ://www.iqvia.com/-/media/iqvia/pdfs/library/publications/acts2018-32nd-edition.pdf?_=1566977658185. Accessed 28 Aug 2019.

22. Bailie GR, Clark JA, Lane CE, Lane PL. Hypersensitivity reactions and deaths associated with intravenous iron preparations. Nephrol Dial Transplant. 2005;20:1443-9. https://doi. org/10.1093/ndt/gfh820.

23. Benoist Bd. Worldwide prevalence of anaemia 1993-2005 of: WHO global database of anaemia. Geneva: World Health Organization; 2008.

24. Moulis G, Sommet A, Durrieu G, Bagheri H, Lapeyre-Mestre M, Montastruc J-L. Trends of reporting of 'serious' vs. 'non-serious' adverse drug reactions over time: a study in the French PharmacoVigilance Database. Br J Clin Pharmacol. 2012;74:201-4. https ://doi.org/10.1111/j.1365-2125.2012.04185.x.

25. Hazell L, Shakir SAW. Under-reporting of adverse drug reactions: a systematic review. Drug Saf. 2006;29:385-96.

26. Arora A, Jalali RK, Vohora D. Relevance of the Weber effect in contemporary pharmacovigilance of oncology drugs. Ther Clin Risk Manag. 2017;13:1195-203. https://doi.org/10.2147/TCRM. S137144.

27. Hoffman KB, Dimbil M, Erdman CB, Tatonetti NP, Overstreet BM. The Weber effect and the United States Food and Drug Administration's Adverse Event Reporting System (FAERS): analysis of sixty-two drugs approved from 2006 to 2010. Drug Saf. 2014;37:283-94. https://doi.org/10.1007/s40264-014-0150-2. 